\title{
Learning Label Preferences: Ranking Error versus Position Error
}

\author{
Eyke Hüllermeier ${ }^{1}$ and Johannes Fürnkranz ${ }^{2}$ \\ 1 Fakultät für Informatik \\ Otto-von-Guericke-Universität Magdeburg, Germany \\ eyke.huellermeier@iti.cs. uni-magdeburg. de \\ 2 Fachbereich Informatik \\ TU Darmstadt, Germany \\ juffi@ke.informatik.tu-darmstadt.de
}

\begin{abstract}
We consider the problem of learning a ranking function, that is a mapping from instances to rankings over a finite number of labels. Our learning method, referred to as ranking by pairwise comparison (RPC), first induces pairwise order relations from suitable training data, using a natural extension of so-called pairwise classification. A ranking is then derived from a set of such relations by means of a ranking procedure. This paper elaborates on a key advantage of such a decomposition, namely the fact that our learner can be adapted to different loss functions by using different ranking procedures on the same underlying order relations. In particular, the Spearman rank correlation is minimized by using a simple weighted voting procedure. Moreover, we discuss a loss function suitable for settings where candidate labels must be tested successively until a target label is found, and propose a ranking procedure for minimizing the corresponding risk.
\end{abstract}

\section{Introduction}

Prediction problems involving complex outputs and structured output spaces have recently received a great deal of attention within the machine learning literature (e.g., [11]). Problems of that kind are particularly challenging, since the prediction of complex structures such as, say, graphs or trees, is more demanding than the prediction of single values as in classification and regression.

A common problem of this type is preference learning, the learning with or from preferences. ${ }^{3}$ In the literature, we can identify two different learning scenarios for preference learning [8]: (i) learning from object preferences, where the task is to order a set of objects according to training information that specifies the preference relations between a set of training objects (see, e.g., [2]), and (ii) learning from label preferences, where the task is to learn a mapping from instances to rankings (total orders) over a finite number of class labels [7]. A

\footnotetext{
${ }^{3}$ Space restrictions prevent a thorough review of related work in this paper, but we refer to [6] and recent workshops in this area, e.g., those at NIPS-02, KI-03, SIGIR03, NIPS-04, and GfKl-05 (the second and fifth organized by the authors).
} 
corresponding ranking function can be seen as an extension of a standard classification function that maps instances to single class labels. In this paper, we focus on the second scenario, but our results can be carried over to the first scenario as well.

In [7], we have introduced a method for learning label preferences that we shall subsequently refer to as ranking by pairwise comparison (RPC). This method works in two phases. First, pairwise order relations (preferences) are learned from suitable training data, using a natural extension of so-called pairwise classification. Then, a ranking is derived from a set of such orders (preferences) by means of a ranking procedure.

The goal of this paper is to show that by using suitable ranking functions, our approach can easily be customized to different performance tasks, that is, to different loss functions for rankings. In fact, the need for a ranking of class labels may arise in different learning scenarios. In this work, we are particularly interested in two types of practically motivated learning problems, one in which the complete ranking is relevant and one in which the predicted ranking has the purpose of reducing the search effort for finding the single target label.

The remainder of the paper is organized as follows: The problem of preference learning is formally introduced in Section 2, and our pairwise approach is presented in Section 3. In Section 4, the aforementioned types of learning problems are discussed and compared in more detail. The ranking procedures suitable for the two types of problems are then discussed in Sections 5 and 6 , respectively.

\section{Learning from Label Preferences}

We consider the following learning problem [8]:

\section{Given:}

- a set of labels $\mathcal{L}=\left\{\lambda_{\imath} \mid \imath=1 \ldots m\right\}$

- a set of examples $\mathcal{S}=\left\{x_{k} \mid k=1 \ldots n\right\}$

- for each training example (instance) $x_{k}$ :

- a set of preferences $P_{k} \subseteq \mathcal{L} \times \mathcal{L}$, where $\left(\lambda_{\imath}, \lambda_{\jmath}\right) \in P_{k}$ indicates that label $\lambda_{\imath}$ is preferred over label $\lambda_{\jmath}$ for instance $x_{k}$.

Find: a function that orders the labels $\lambda \in \mathcal{L}$ for any given example.

We will abbreviate $\left(\lambda_{\imath}, \lambda_{\jmath}\right) \in P_{k}$ with $\lambda_{\imath} \succ_{x_{k}} \lambda_{\jmath}$ or simply $\lambda_{\imath} \succ \lambda_{\jmath}$ if the particular example $x_{k}$ doesn't matter or is clear from the context.

The above setting has recently been introduced as constraint classification in [9]. As shown in that paper, it is a generalization of several common learning settings, in particular

- ranking: Each training example is associated with a total order of the labels.

- classification: A single class label $\lambda_{x}$ is assigned to each example $x$; implicitly, this defines the set of preferences $\left\{\lambda_{x} \succ \lambda \mid \lambda \in \mathcal{L} \backslash\left\{\lambda_{x}\right\}\right\}$. 
- multi-label classification: Each example $x$ is associated with a subset $L_{x} \subseteq \mathcal{L}$ of labels; implicitly, this defines the preferences $\left\{\lambda \succ \lambda^{\prime} \mid \lambda \in L_{x}, \lambda^{\prime} \in \mathcal{L} \backslash L_{x}\right\}$.

As mentioned above, we are mostly interested in the first problem, that is in predicting a ranking (complete, transitive, asymmetric relation) of the labels. The ranking $\succ_{x}$ of an instance $x$ can be expressed in terms of a permutation $\tau_{x}$ of $\{1 \ldots m\}$ such that

$$
\lambda_{\tau_{x}(1)} \succ_{x} \lambda_{\tau_{x}(2)} \succ_{x} \ldots \succ_{x} \lambda_{\tau_{x}(m)}
$$

Note that we make the simplifying assumption that all preferences are strict, i.e., we do not consider the case of indifference between labels.

An appealing property of this learning framework is that its input, consisting of comparative preference information of the form $\lambda_{\imath} \succ_{x} \lambda_{\jmath}\left(x\right.$ prefers $\lambda_{\imath}$ to $\left.\lambda_{\jmath}\right)$, is often easier to obtain than absolute ratings of single alternatives in terms of utility degrees. In this connection, note that knowledge about the complete ranking (1) can be expanded into $m(m-1) / 2$ comparative preferences $\lambda_{\tau_{x}(\imath)} \succ$ $\lambda_{\tau_{x}(\jmath)}, 1 \leq \imath<\jmath \leq m$.

\section{Learning Pairwise Preferences}

The idea of pairwise learning is well-known in the context of classification [5], where it allows one to transform a multi-class classification problem, i.e., a problem involving $m>2$ classes $\mathcal{L}=\left\{\lambda_{1} \ldots \lambda_{m}\right\}$, into a number of binary problems. To this end, a separate model (base learner) $\mathcal{M}_{\imath \jmath}$ is trained for each pair of labels $\left(\lambda_{\imath}, \lambda_{\jmath}\right) \in \mathcal{L}, 1 \leq \imath<\jmath \leq m$; thus, a total number of $m(m-1) / 2$ models is needed. $\mathcal{M}_{\imath \jmath}$ is intended to separate the objects with label $\lambda_{\imath}$ from those having label $\lambda_{\jmath}$.

At classification time, a query $x$ is submitted to all learners, and each prediction $\mathcal{M}_{\imath \jmath}(x)$ is interpreted as a vote for a label. If classifier $\mathcal{M}_{\imath \jmath}$ predicts $\lambda_{\imath}$, this is counted as a vote for $\lambda_{\imath}$. Conversely, the prediction $\lambda_{\jmath}$ would be considered as a vote for $\lambda_{\jmath}$. The label with the highest number of votes is then proposed as a prediction.

The above procedure can be extended to the case of preference learning in a natural way [7]. A preference information of the form $\lambda_{\imath} \succ_{x} \lambda_{\jmath}$ is turned into a training example $(x, y)$ for the learner $\mathcal{M}_{a b}$, where $a=\min (\imath, \jmath)$ and $b=\max (\imath, \jmath)$. Moreover, $y=1$ if $\imath<\jmath$ and $y=0$ otherwise. Thus, $\mathcal{M}_{a b}$ is intended to learn the mapping that outputs 1 if $\lambda_{a} \succ_{x} \lambda_{b}$ and 0 if $\lambda_{b} \succ_{x} \lambda_{a}$ :

$$
x \mapsto\left\{\begin{array}{lll}
1 & \text { if } & \lambda_{a} \succ_{x} \lambda_{b} \\
0 & \text { if } & \lambda_{b} \succ_{x} \lambda_{a}
\end{array} .\right.
$$

The mapping (2) can be realized by any binary classifier. Alternatively, one might of course employ a classifier that maps into the unit interval $[0,1]$ instead of $\{0,1\}$. The output of such a "soft" binary classifier can usually be interpreted as a probability or, more generally, a kind of confidence in the classification. 
Thus, the closer the output of $\mathcal{M}_{a b}$ to 1 , the stronger the preference $\lambda_{a} \succ_{x} \lambda_{b}$ is supported.

A preference learner composed of an ensemble of soft binary classifiers (which can be constructed on the basis of training data in the form of instances with associated partial preferences) assigns a valued preference relation $\mathcal{R}_{x}$ to any (query) instance $x \in \mathcal{X}$ :

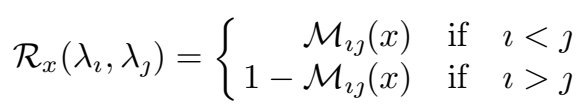

for all $\lambda_{\imath} \neq \lambda_{\jmath} \in \mathcal{L}$.

Given a preference relation $\mathcal{R}_{x}$ for an instance $x$, the next question is how to derive an associated ranking $\tau_{x}$. This question is non-trivial, since a relation $\mathcal{R}_{x}$ does not always suggest a unique ranking in an unequivocal way. In fact, the problem of inducing a ranking from a (valued) preference relation has received a lot of attention in several research fields, e.g., in fuzzy preference modeling and (multi-attribute) decision making [4]. Besides, in the context of our application, it turned out that the ranking procedure used to transform a relation $\mathcal{R}_{x}$ into a ranking $\tau_{x}$ is closely related to the definition of the quality of a prediction and, hence, to the intended purpose of a ranking. In other words, risk minimization with respect to different loss functions might call for different ranking procedures.

\section{Ranking Error versus Position Error}

In Section 2, we introduced the problem of predicting a ranking of class labels in a formal way, but did not discuss the semantics of a predicted ranking. In fact, one should realize that such a ranking can serve different purposes. Needless to say, this point is of major importance for the evaluation of a predicted ranking.

In this paper, we are especially interested in two types of practically motivated performance tasks. In the first setting, which is probably the most obvious one, the complete ranking is relevant, i.e., the positions assigned to all of the labels. As an example, consider the problem to order the questions in a questionnaire. Here, the goal is to maximize a particular respondents' motivation to complete the questionnaire. Another example is learning to predict the best order in which to supply a certain set of stores (route of a truck), depending on external conditions like traffic, weather, purchase order quantities, etc.

In case the complete ranking is relevant, the quality of a prediction should be quantified in terms of a distance measure between the predicted and the true ranking. We shall refer to any deviation of the predicted ranking from the true one as a ranking error.

To motivate the second setting, consider a fault detection problem which consists of identifying the cause for the malfunctioning of a technical system. If it turned out that a predicted cause is not correct, an alternative candidate must be tried. A ranking then suggests a simple (trial and error) search process, which successively tests the candidates, one by one, until the correct cause is found [1]. In this scenario, where labels correspond to causes, the existence of a 
single target label (instead of a target ranking) is assumed. Hence, an obvious measure of the quality of a predicted ranking is the number of futile trials made before that label is found. A deviation of the predicted target label's position from the top-rank will subsequently be called a position error.

The main difference between the two types of error is that an evaluation of a full ranking (ranking error) attends to all positions. For example, if the two highest ranks of the true ranking are swapped in the predicted ranking, this is as bad as the swapping of the two lowest ranks.

Note that the position error is closely related to the conventional (classification) error, i.e., the incorrect prediction of the top label. In both cases, we are eventually concerned with predictions for the top rank. In our setting, however, we not only try to maximize the number of correct predictions. Instead, in the case of a misclassification, we also look at the position of the target label. The higher this position, the better the prediction. In other words, we differentiate between "bad" predictions in a more subtle way.

Even though we shall not deepen this point in the current paper, we note that the idea of a position error can of course be generalized to multi-label (classification) problems which assume several instead of a single target label for each instance. There are different options for such a generalization. For example, it makes a great difference whether one is interested in having at least one of the targets on a top rank (e.g., since one solution is enough), or whether all of them should have high positions (resp. none of them should be ranked low). An application of the latter type has recently been studied in [3].

\section{Minimizing the Ranking Error}

The quality of a model $\mathcal{M}$ (induced by a learning algorithm) is commonly expressed in terms of its expected loss or risk

$$
\mathbb{E}(D(y, \mathcal{M}(x))),
$$

where $D(\cdot)$ is a loss or distance function, $\mathcal{M}(x)$ denotes the prediction made by the learning algorithm for the instance $x$, and $y$ is the true outcome. The expectation $\mathbb{E}$ is taken over $\mathcal{X} \times \mathcal{Y}$, where $\mathcal{Y}$ is the output space (e.g., the set $\mathcal{L}$ of classes in classification). ${ }^{4}$

The simplest loss function, commonly employed in classification, is the $0 / 1-$ loss: $D(y, \hat{y})=0$ for $y=\hat{y}$ and $=1$ otherwise. Given this loss function, the optimal (Bayes) prediction for a specific instance $x$ is simply the most probable outcome $y$. In the classification setting, for example, where $\mathcal{Y}=\mathcal{L}$, this estimate is the class with maximum posterior probability $\mathrm{P}\left(\lambda_{\imath} \mid x\right)$.

A straightforward generalization of this principle to the ranking setting, where $\mathcal{Y}$ is the class of rankings over $\mathcal{L}$, leads to the prediction

$$
\hat{\tau}_{x}=\arg \max _{\tau \in \mathcal{S}_{m}} \mathrm{P}(\tau \mid x),
$$

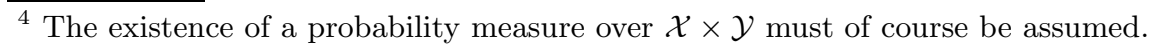


where $\mathrm{P}(\tau \mid x)$ is the conditional probability of a ranking (permutation) given an instance $x$, and $\mathcal{S}_{m}$ denotes the class of all permutations of $\{1 \ldots m\}$.

Obviously, the simple 0/1-distance function is a rather crude evaluation measure for rankings, because it assigns the same loss to all rankings that differ from the correct ranking, and does not take into account that different rankings can have different degrees of similarity. For this reason, a number of more sophisticated distance measures for rankings have been proposed in literature.

In general, if $D\left(\tau, \tau^{\prime}\right)$ is a measure of the distance between two rankings $\tau$ and $\tau^{\prime}$, the risk minimizing prediction is

$$
\hat{\tau}_{x}=\arg \min _{\tau \in S_{k}} \sum_{\tau \in \mathcal{S}_{m}} D\left(\tau, \tau^{\prime}\right) \cdot \mathrm{P}\left(\tau^{\prime} \mid x\right) .
$$

A frequently used distance measure is the sum of squared rank distances

$$
D\left(\tau^{\prime}, \tau\right) \stackrel{\text { df }}{=} \sum_{\imath=1}^{m}\left(\tau^{\prime}(\imath)-\tau(\imath)\right)^{2}
$$

which is equivalent to the Spearman rank correlation $^{5}$

$$
1-\frac{6 D\left(\tau, \tau^{\prime}\right)}{m\left(m^{2}-1\right)} \in[-1,1]
$$

$\mathrm{RPC}$ can yield a risk minimizing prediction for this loss function, if the predictions of the binary classifiers are combined by weighted voting, i.e., the alternatives $\lambda_{\imath}$ are evaluated by means of the sum of weighted votes

$$
S\left(\lambda_{\imath}\right)=\sum_{\lambda_{\jmath} \neq \lambda_{\imath}} \mathcal{R}_{x}\left(\lambda_{\imath}, \lambda_{\jmath}\right)
$$

and ranked according to these evaluations:

$$
\lambda_{\tau_{x}(1)} \succ_{x} \lambda_{\tau_{x}(2)} \succ_{x} \ldots \succ_{x} \lambda_{\tau_{x}(m)}
$$

with $\tau_{x}$ satisfying $S\left(\lambda_{\tau_{x}(\imath)}\right) \geq S\left(\lambda_{\tau_{x}(\imath+1)}\right), \imath=1 \ldots m-1 .{ }^{6}$ This is a particular type of "ranking by scoring" strategy; here, the scoring function is given by (6).

Formally, we can show the following result, which provides a theoretical justification for the voting procedure (6). The proof of this theorem can be found in Appendix A.

Theorem 1. Using the "ranking by scoring" procedure outlined above, RPC is a risk minimizer with respect to (5) as a loss function. More precisely, with

$$
\mathcal{M}_{\imath \jmath}(x)=\mathrm{P}\left(\lambda_{\imath} \succ_{x} \lambda_{\jmath}\right)=\sum_{\tau: \tau(\jmath)<\tau(\imath)} \mathrm{P}(\tau \mid x),
$$

\footnotetext{
${ }^{5}$ This is, of course, a similarity rather than a distance measure.

6 Ties can be broken arbitrarily.
} 
the expected distance

$$
E\left(\tau^{\prime}\right)=\sum_{\tau} p(\tau) \cdot D\left(\tau^{\prime}, \tau\right)=\sum_{\tau} p(\tau) \sum_{\imath=1}^{m}\left(\tau^{\prime}(\imath)-\tau(\imath)\right)^{2}
$$

becomes minimal by choosing $\tau^{\prime}$ such that $\tau^{\prime}(\imath) \leq \tau^{\prime}(\jmath)$ whenever $S\left(\lambda_{\imath}\right) \geq S\left(\lambda_{\jmath}\right)$, where $S\left(\lambda_{\imath}\right)$ is given by (6).

\section{Minimizing the Position Error}

Despite the fact that (5) is a reasonable loss function for rankings, it is not always appropriate. In particular, it assumes that the complete ranking is relevant for the quality of a prediction, which is not the case in connection with the fault detection scenario outlined in the introduction. Here, only the prefix of a ranking $\tau_{x}$ is considered, up to the position of the target label $\lambda_{x}$, while the rest of the prediction is of no importance (since the search procedure stops if $\lambda_{x}$ has been found). In this case, the loss function only depends on the rank of $\lambda_{x}$.

More specifically, we define the position error as $\tau_{x}^{-1}\left(\lambda_{x}\right)$, i.e., by the position of the target label $\lambda_{x}$ in the ranking $\tau_{x}$. To compare the quality of rankings of different problems, it is useful to normalize the position error for the number of labels. This normalized position error is defined as

$$
\frac{\tau_{x}^{-1}\left(\lambda_{x}\right)-1}{m-1} \in\{0,1 /(m-1) \ldots 1\},
$$

What kind of ranking procedure should be used in order to minimize the risk of a predicted ranking with respect to the position error as a loss function? Intuitively, the candidate labels $\lambda$ should now be ordered according to their probability $\mathrm{P}\left(\lambda=\lambda_{x}\right)$ of being the target label. Especially, the top-rank (first position) should be given to the label $\lambda_{\top}$ for which this probability is maximal. Regarding the second rank, recall the fault detection metaphor, where the second hypothesis for the cause of the fault is only tested in case the first one turned out to be wrong. In this setting, the second rank should not simply be given to the label with the second highest probability according to the measure $\mathrm{P}_{1}(\cdot)=$ $\mathrm{P}(\cdot)$. Instead, it must be assigned to the label that maximizes the conditional probability $\mathrm{P}_{2}(\cdot)=\mathrm{P}\left(\cdot \mid \lambda_{x} \neq \lambda_{\top}\right)$, i.e., the probability of being the target label given that the first proposal was incorrect.

At first sight, passing from $\mathrm{P}_{1}(\cdot)$ to $\mathrm{P}_{2}(\cdot)$ might appear meaningless from a ranking point of view, since standard probabilistic conditioning (dividing all probabilities by $1-\mathrm{P}\left(\lambda_{\top}\right)$ and setting $\mathrm{P}\left(\lambda_{\top}\right)=0$ ) does not change the order of the remaining labels. One should realize, however, that standard conditioning is not an incontestable updating procedure in our context, simply because $\mathrm{P}_{1}(\cdot)$ is not a "true" measure over the class labels. Rather, it is only an estimated measure coming from a learning algorithm. Thus, it seems sensible to perform "conditioning" not on the measure itself, but rather on the learner that produced the measure. By this we mean retraining the learner on the original data 
without the $\lambda_{T}$-examples, something that could be paraphrased as "empirical conditioning". To emphasize that this type of conditioning depends on the data $\mathcal{D}$ and the model assumptions (hypothesis space) $\mathcal{H}$ and, moreover, that it concerns an estimated ("hat") probability, the conditional measure $\mathrm{P}_{2}(\cdot)$ could be written more explicitly as

$$
\mathrm{P}_{2}(\cdot)=\widehat{\mathrm{P}}\left(\cdot \mid \lambda_{x} \neq \lambda_{\mathrm{T}}, \mathcal{D}, \mathcal{M}\right) .
$$

To motivate the idea of empirical conditioning, suppose that the estimated probabilities come from a classification tree. Of course, the original tree trained with the complete data will be highly influenced by $\lambda_{T \text {-examples, and the proba- }}$ bilities assigned by that tree to the alternatives $\lambda \neq \lambda_{\top}$ might be inaccurate. Retraining a classification tree on a reduced set of data might then lead to more accurate probabilities for the remaining labels, especially since the multi-class problem to be solved has now become simpler (as it involves fewer classes).

A problem of the above "ranking through iterated choice" procedure, that is, the successive selection of alternatives by estimating top-labels from (conditional) probability measures $\mathrm{P}_{1}(\cdot), \mathrm{P}_{2}(\cdot) \ldots \mathrm{P}_{m}(\cdot)$, concerns its computational complexity. In fact, realizing empirical conditioning by retraining a standard multi-class classifier comes down to training such a classifier for (potentially) each subset of the label set $\mathcal{L}$. Fortunately, empirical conditioning can be implemented much more efficiently by our pairwise approach, as will now be shown.

\subsection{Implementing "ranking through iterated choice" by RPC}

What kind of aggregation procedure is suitable for deriving an estimated probability distribution from pairwise classifications resp. valued preference $\mathcal{R}\left(\lambda_{l}, \lambda_{\jmath}\right)$ ? Let $E_{\imath}$ denote the event that $\lambda_{\imath}=\lambda_{x}$, i.e., that $\lambda_{\imath}$ is the target label, and let $E_{\imath \jmath}=E_{\imath} \vee E_{\jmath}$ (either $\lambda_{\imath}$ or $\lambda_{\jmath}$ is the target). Then,

$$
(m-1) \mathrm{P}\left(E_{\imath}\right)=\sum_{\jmath \neq \imath} \mathrm{P}\left(E_{\imath}\right)=\sum_{\jmath \neq \imath} \mathrm{P}\left(E_{\imath} \mid E_{\imath \jmath}\right) \mathrm{P}\left(E_{\imath \jmath}\right),
$$

where $m$ is the number of labels. Considering the (pairwise) estimates $\mathcal{R}\left(\lambda_{\imath}, \lambda_{\jmath}\right)$ as conditional probabilities $\mathrm{P}\left(E_{\imath} \mid E_{\imath}\right)$, we obtain a system of linear equations for the (unconditional) probabilities $\mathrm{P}\left(E_{\imath}\right)$ :

$$
\begin{aligned}
\mathrm{P}\left(E_{\imath}\right) & =\frac{1}{m-1} \sum_{\jmath \neq \imath} \mathcal{R}\left(\lambda_{\imath}, \lambda_{\jmath}\right) \mathrm{P}\left(E_{\imath \jmath}\right) \\
& =\frac{1}{m-1} \sum_{\jmath \neq \imath} \mathcal{R}\left(\lambda_{\imath}, \lambda_{\jmath}\right)\left(\mathrm{P}\left(E_{\imath}\right)+\mathrm{P}\left(E_{\jmath}\right)\right)
\end{aligned}
$$

In conjunction with the constraint $\sum_{\imath=1}^{m} \mathrm{P}\left(E_{\imath}\right)=1$, this system has a unique solution provided that $\mathcal{R}\left(\lambda_{\imath}, \lambda_{\jmath}\right)>0$ for all $1 \leq \imath, \jmath \leq m[12]$.

Based on this result, the "ranking through iterated choice" procedure suggested above can be realized as follows: First, the system of linear equations 
(10) is solved and the label $\lambda_{\imath}$ with maximal probability $\mathrm{P}\left(E_{\imath}\right)$ is chosen as the top-label $\lambda_{\top}$. This label is then removed, i.e., the corresponding row and column of the relation $\mathcal{R}$ is deleted. To find the second best label, the same procedure is then applied to the reduced relation, i.e., by solving a system of $m-1$ linear equations. This process is iterated until a full ranking has been constructed.

Lemma 1. In each iteration of the above "ranking through iterated choice" procedure, the correct conditional probabilities are derived.

Proof. Without loss of generality, assume that $\lambda_{m}$ has obtained the highest rank in the first iteration. The information that this label is incorrect, $\lambda_{m} \neq \lambda_{x}$, is equivalent to $\mathrm{P}\left(E_{m}\right)=0, \mathrm{P}\left(E_{m} \mid E_{\jmath m}\right)=0$, and $\mathrm{P}\left(E_{\jmath} \mid E_{\jmath m}\right)=1$ for all $\jmath \neq m$. Incorporating these probabilities in (10) yields, for all $\imath<m$,

$$
\begin{aligned}
(m-1) \mathrm{P}\left(E_{\imath}\right) & =\sum_{\jmath=1 \ldots m, \jmath \neq \imath} \mathrm{P}\left(E_{\imath} \mid E_{\imath \jmath}\right) \mathrm{P}\left(E_{\imath \jmath}\right) \\
& =\sum_{\jmath=1 . . m-1, \jmath \neq \imath} \mathrm{P}\left(E_{\imath} \mid E_{\imath \jmath}\right) \mathrm{P}\left(E_{\imath \jmath}\right)+1 \mathrm{P}\left(E_{\imath m}\right)
\end{aligned}
$$

and as $\mathrm{P}\left(E_{\imath m}\right)=\mathrm{P}\left(E_{\imath}\right)+\mathrm{P}\left(E_{m}\right)=\mathrm{P}\left(E_{\imath}\right)$,

$$
(m-2) \mathrm{P}\left(E_{\imath}\right)=\sum_{\jmath=1 . . m-1, \jmath \neq \imath} \mathrm{P}\left(E_{\imath} \mid E_{\imath \jmath}\right) \mathrm{P}\left(E_{\imath \jmath}\right) .
$$

Obviously, the last equation is equivalent to (10) for a system with $m-1$ labels, namely the system obtained by removing the $m$-th row and column of $\mathcal{R}$.

As can be seen, the pairwise approach is particularly well-suited for the "ranking through iterated choice" procedure, as it allows for an easy incorporation of the information coming from futile trials. One just has to solve the system of linear equations (10) once more, with some of the pairwise probabilities set to 0 resp. 1 (or, equivalently, solve a smaller system of equations). No retraining of any classifier is required!

Theorem 2. By ranking the alternative labels according to their (conditional) probabilities of being the top-label, RPC becomes a risk minimizer with respect to the position error (8) as a loss function. That is, the expected loss

$$
E(\tau)=\frac{1}{m-1} \sum_{\imath=1}^{m}(\imath-1) \cdot \mathrm{P}\left(\lambda_{\tau(\imath)}=\lambda_{x}\right)
$$

becomes minimal for the ranking predicted by $R P C$.

Proof. This result follows almost by definition. In fact, note that we have

$$
E(\tau) \propto \sum_{\imath=1}^{m} \mathrm{P}\left(\lambda_{x} \notin\left\{\lambda_{\tau(1)} \ldots \lambda_{\tau(\imath)}\right\}\right),
$$

and that, for each position $\imath$, the probability to excess this position when searching for the target $\lambda_{x}$ is obviously minimized when ordering the labels according to their (conditional) probabilities. 


\section{Concluding Remarks}

By showing that RPC is a risk minimizer with respect to particular loss functions for rankings, this paper provides a sound theoretical foundation for our method of ranking by pairwise comparison. The interesting point is that RPC can easily be customized to different performance tasks, simply by changing the ranking procedure employed in the second step of the method. By modifying this procedure, the goal of RPC can be changed from minimizing the expected distance between the predicted and the true ranking to minimizing the expected number of futile trials in searching a target label. This can be done without retraining of the classifier ensemble.

Apart from these theoretical results, the practical validation of our method is of course an important issue. Regarding the ranking error, RPC has already been investigated empirically in $[7,10]$, whereas empirical studies concerning the position error constitute a topic of still ongoing work. In this context, it is particularly interesting to compare the results obtained by the "ranking through iterated choice" procedure with predictions from standard ("non-iterated") probabilistic classification.

\section{References}

1. C. Alonso, JJ. Rodríguez, and B. Pulido. Enhancing consistency based diagnosis with machine learning techniques. In Current Topics in AI, vol. 3040 of LNAI, 312-321. Springer, 2004.

2. W.W. Cohen, R.E. Schapire, and Y. Singer. Learning to order things. Journal of Artificial Intelligence Research, 10:243-270, 1999.

3. K. Crammer and Y. Singer. A family of additive online algorithms for category ranking. Journal of Machine Learning Research, 3:1025-1058, 2003.

4. J. Fodor and M. Roubens. Fuzzy Preference Modelling and Multicriteria Decision Support. Kluwer, 1994.

5. J. Fürnkranz. Round robin classification. Journal of Machine Learning Research, 2:721-747, 2002.

6. J. Fürnkranz and E. Hüllermeier. Pairwise preference learning and ranking. Technical Report TR-2003-14, Österr. Forschungsinst. für Artif. Intell. Wien, 2003.

7. J. Fürnkranz and E. Hüllermeier. Pairwise preference learning and ranking. In Proc. ECML-03, Cavtat-Dubrovnik, Croatia, September 2003. Springer-Verlag.

8. J. Fürnkranz and E. Hüllermeier. Preference learning. Künstliche Intelligenz, 1/05:60-61, 2005.

9. S. Har-Peled, D. Roth, and D. Zimak. Constraint classification: a new approach to multiclass classification. In Proc. ALT-02, pp. 365-379, Lübeck, 2002. Springer.

10. E. Hüllermeier and J. Fürnkranz. Comparison of ranking procedures in pairwise preference learning. In Proc. 10th Int. Conf. Information Processing and Management of Uncertainty in Knowledge-Based Systems (IPMU-04), Perugia, 2004.

11. I. Tsochantaridis, T. Hofmann, T. Joachims, and Y. Altun. Support vector machine learning for interdependent and structured output spaces. In Proc. 21st Int. Conf. on Machine Learning (ICML-2004), pp. 823-830, Banff, Alberta, Canada, 2004.

12. T.F. Wu, C.J. Lin, and R.C. Weng. Probability estimates for multi-class classification by pairwise coupling. J. of Machine Learning Research, 5:975-1005, 2004. 


\section{A Proof of Theorem 1}

Lemma 1: Let $s_{\imath}, \imath=1 \ldots m$, be real numbers such that $0 \leq s_{1} \leq s_{2} \ldots \leq s_{m}$. Then, for all permutations $\tau \in \mathcal{S}_{m}$,

$$
\sum_{\imath=1}^{m}\left(\imath-s_{\imath}\right)^{2} \leq \sum_{\imath=1}^{m}\left(\imath-s_{\tau(\imath)}\right)^{2}
$$

Proof. We have

$$
\begin{aligned}
\sum_{\imath=1}^{m}\left(\imath-s_{\tau(\imath)}\right)^{2} & =\sum_{\imath=1}^{m}\left(\imath-s_{\imath}+s_{\imath}-s_{\tau(\imath)}\right)^{2} \\
& =\sum_{\imath=1}^{m}\left(\imath-s_{\imath}\right)^{2}+2 \sum_{\imath=1}^{m}\left(\imath-s_{\imath}\right)\left(s_{\imath}-s_{\tau(\imath)}\right)+\sum_{\imath=1}^{m}\left(s_{\imath}-s_{\tau(\imath)}\right)^{2} .
\end{aligned}
$$

Expanding the last equation and exploiting that $\sum_{\imath=1}^{m} s_{\imath}^{2}=\sum_{\imath=1}^{m} s_{\tau(\imath)}^{2}$ yields

$$
\sum_{\imath=1}^{m}\left(\imath-s_{\tau(\imath)}\right)^{2}=\sum_{\imath=1}^{m}\left(\imath-s_{\imath}\right)^{2}+2 \sum_{\imath=1}^{m} \imath s_{\imath}-2 \sum_{\imath=1}^{m} \imath s_{\tau(\imath)} .
$$

On the right-hand side of the last equation, only the last term $\sum_{\imath=1}^{m} \imath s_{\tau(\imath)}$ depends on $\tau$. Since $s_{\imath} \leq s_{\jmath}$ for $\imath<\jmath$, this term becomes maximal for $\tau(\imath)=\imath$. Therefore, the right-hand side is larger than or equal to $\sum_{\imath=1}^{m}\left(\imath-s_{\imath}\right)^{2}$, which proves the lemma.

Lemma 2. Let $\mathrm{P}(\cdot \mid x)$ be a probability distribution over $\mathcal{S}_{m}$ and let $p(\tau) \stackrel{\mathrm{df}}{=}$ $\mathrm{P}(\tau \mid x)$. Moreover, let

$$
s_{\imath} \stackrel{\mathrm{df}}{=} m-\sum_{\jmath \neq \imath} \mathrm{P}\left(\lambda_{\imath} \succ_{x} \lambda_{\jmath}\right)
$$

with

$$
\mathrm{P}\left(\lambda_{\iota} \succ_{x} \lambda_{\jmath}\right)=\sum_{\tau: \tau(\jmath)<\tau(\imath)} \mathrm{P}(\tau \mid x) .
$$

Then, $s_{\imath}=\sum_{\jmath \neq \imath} p(\tau) \tau(\imath)$.

Proof. We have

$$
\begin{aligned}
s_{\imath} & =m-\sum_{\jmath \neq \imath} \mathrm{P}\left(\lambda_{\imath} \succ_{x} \lambda_{\jmath}\right)=1+\sum_{\jmath \neq \imath}\left(1-\mathrm{P}\left(\lambda_{\imath} \succ_{x} \lambda_{\jmath}\right)\right) \\
& =1+\sum_{\jmath \neq \imath} \mathrm{P}\left(\lambda_{\jmath} \succ_{x} \lambda_{\imath}\right)=1+\sum_{\jmath \neq \imath} \sum_{\tau: \tau(\jmath)<\tau(\imath)} p(\tau) \\
& =1+\sum_{\tau} p(\tau) \sum_{\jmath \neq \imath}\left\{\begin{array}{lll}
1 & \text { if } & \tau(\imath)>\tau(\jmath) \\
0 & \text { if } & \tau(\imath)<\tau(\jmath)
\end{array}\right. \\
& =1+\sum_{\tau} p(\tau)(\tau(\imath)-1)=\sum_{\tau} p(\tau) \tau(\imath)
\end{aligned}
$$


Under the assumption that the base learners' estimates correspond exactly to the probabilities of pairwise preference, i.e.,

$$
\mathcal{R}_{x}\left(\lambda_{\imath}, \lambda_{\jmath}\right)=\mathcal{M}_{\imath \jmath}(x)=\mathrm{P}\left(\lambda_{\imath} \succ_{x} \lambda_{\jmath}\right),
$$

$s_{\imath} \leq s_{\jmath}$ is equivalent to $S\left(\lambda_{\imath}\right) \geq S\left(\lambda_{\jmath}\right)$. Thus, ranking the alternatives according to $S\left(\lambda_{\imath}\right)$ (in decreasing order) is equivalent to ranking them according to $s_{\imath}$ (in increasing order).

Theorem 1. The expected distance

$$
E\left(\tau^{\prime}\right)=\sum_{\tau} p(\tau) \cdot D\left(\tau^{\prime}, \tau\right)=\sum_{\tau} p(\tau) \sum_{\imath=1}^{m}\left(\tau^{\prime}(\imath)-\tau(\imath)\right)^{2}
$$

becomes minimal by choosing $\tau^{\prime}$ such that $\tau^{\prime}(\imath) \leq \tau^{\prime}(\jmath)$ whenever $s_{\imath} \leq s_{\jmath}$, with $s_{\imath}$ given by (12).

Proof. We have

$$
\begin{aligned}
E\left(\tau_{x}^{\prime}\right)= & \sum_{\tau} p(\tau) \sum_{\imath=1}^{m}\left(\tau_{x}^{\prime}(\imath)-\tau(\imath)\right)^{2} \\
= & \sum_{\imath=1}^{m} \sum_{\tau} p(\tau)\left(\tau_{x}^{\prime}(\imath)-\tau(\imath)\right)^{2} \\
= & \sum_{\imath=1}^{m} \sum_{\tau} p(\tau)\left(\tau_{x}^{\prime}(\imath)-s_{\imath}+s_{\imath}-\tau(\imath)\right)^{2} \\
= & \sum_{\imath=1}^{m} \sum_{\tau} p(\tau)\left[\left(\tau(\imath)-s_{\imath}\right)^{2}-2\left(\tau(\imath)-s_{\imath}\right)\left(s_{\imath}-\tau^{\prime}(\imath)\right)\right. \\
& \left.\quad+\left(s_{\imath}-\tau^{\prime}(\imath)\right)^{2}\right] \\
& \sum_{\imath=1}^{m}\left[\sum_{\tau} p(\tau)\left(\tau(\imath)-s_{\imath}\right)^{2}-2\left(s_{\imath}-\tau^{\prime}(\imath)\right) \cdot\right. \\
& \left.\cdot \sum_{\tau} p(\tau)\left(\tau(\imath)-s_{\imath}\right)+\sum_{\tau} p(\tau)\left(s_{\imath}-\tau^{\prime}(\imath)\right)^{2}\right]
\end{aligned}
$$

In the last equation, the mid-term on the right-hand side becomes 0 according to Lemma 2. Moreover, the last term obviously simplifies to $\left(s_{\imath}-\tau^{\prime}(\imath)\right)$, and the first term is a constant $c=\sum_{\tau} p(\tau)\left(\tau(\imath)-s_{\imath}\right)^{2}$ that does not depend on $\tau^{\prime}$. Thus, we obtain $E\left(\tau_{x}^{\prime}\right)=c+\sum_{\imath=1}^{m}\left(s_{\imath}-\tau^{\prime}(\imath)\right)^{2}$ and the theorem follows from Lemma 1. 